\title{
The Effect of Swiss Ball Program on Lower Extremity Function of Elderly with Mild Cognitive Impairment
}

\begin{abstract}
The purpose of this study was to determine the effects of swiss ball program training on lower extremity function of old elderly with mild cognitive impairment. The subjects were 30 mild cognitive impairment eldrly people aged between 65 and 80, who were divided into the swiss ball program training group $(n=15)$ and the control group $(n=15)$. The swiss ball program training group engaged in a 50-minute exercise session using. Lower extremity function scale of the two groups were measured before and after the intervention. The results are as follows. According to the swiss ball program training conducted to examine the effects of the training on lower extremity function, Moreover, the two groups showed significant differences in lower extremity function. These results indicate that virtual reality training is effective in improving lower extremity function. To conclusion, swiss ball program was found to have a positive effect on elderly lower extremity function. Swiss ball program training can be proposed as a form of fall prevention exercise for the mild cognitive impairment. Swiss ball program may be helpful to reduce the incidence of dementia and behavioral complications.
\end{abstract}

Key words: Mild Cognitive Impairment; Lower Extremity Function; Swiss Ball Program
Yu Jin Lee ${ }^{a}$, Jae Young Park ${ }^{b}$, Hyun Jeong Park ${ }^{\mathrm{b}}$, Hee Joon Shin ${ }^{b}$, Duk Kyu Choib, Hyung Soo Shin $^{\mathrm{b}}$

${ }^{a}$ Daegu Catholic University, Daegu; ' ${ }^{\text {Kyungwoon }}$ University, Gumi, Korea

Received : 9 January 2014

Revised : 13 February 2014

Accepted : 28 March 2014

Address for correspondence

Hyung Soo Shin, PT, Ph.D

Department of Physical Therapy, Kyungwoon University, 730, Gangdongro, Sandong-myeon, Gumi, Korea

Tel: 82-54-479-1372

E-mail: hsshin@ikw.ac.kr

\section{INTRODUCTION}

As the increase of the elderly population is emerging as social issues, interests in the quality of life of the elderly are rising in all parts of our society. Raising health awareness of the elderly is becoming an important issue that has to be dealt with by the elderly themselves, their dependents, and also at the national level.

Dementia is a typical organic mental impairment in which cognitive function and higher mental function deteriorate. It can be defined as a complex medical syndrome that is accompanied by memory impairment, behavioral impairment, personality changes, etc. and disrupts not only the everyday life but also professional activities and social life. In particular, as Alzheimer's dementia, having a morbidity rate of $71 \%$ of all senile dementia, is a neurodegenerative disorder that affects everyday life through memory loss and cognitive changes, the efforts to prevent it and its aggravation are required at individual and national levels.

Some studies report lower extremity impairment in the elderly with Alzheimer's dementia and mild cognitive impairment, the stage before dementia. They also report that the lower extremity impairment rate increases with the cognitive function impairment level increased(1, 2). As such drop in mobility of lower extremity can decline the quality of life of the elderly by affecting physical activities and activities of everyday life, recently, the correlation between the physical activities of the elderly and reduction of cognitive function is being studied extensively.

As exercises programs are proven to be affective in improving the activities of everyday living and physical function of the elderly with dementia, studies on exercise therapy are actively being car 
ried out(3). The purpose of the exercise therapy forthe elderly with dementia is improving the quality of life(4). Regular exercises for the dementia patients reduce the incidence of adult diseases and strokes such as diabetes, hyperlipidemia, high blood pressure, obesity, the causes of dementia. They are also effective in reducing the risk of falling by vitalizing brain metabolism, increasing the cerebral blood flow and neurotransmitter secretion and improving the flexibility and balance sense of muscles $(5,6)$.

In some studies, it has been reported that exercises have positive effects on cognitive function. And subsequent studies conducted on elderly recognition and physical activities reported that a 24-week physical exercise intervention improves the elderly cognitive function $(7,8)$. They also reported that the swiss ball program has an effect of motivating the participants by inducing interests and fun. Moreover, as its reliability has been proven, studies related to the swiss ball program are being actively carried out.

Through the results of the preceding studies, it can be expected that active exercises and physical activities of the elderly can significantly improve lower extremity function, a factor that predicts the development and spread of dementia. Therefore, this study, by implementing the swiss ball program on the elderly with mild cognitive impairment, will investigate what effects the swiss ball program has on the cognitive function and lower extremity function.

\section{METHODS}

\section{Subjects}

This study was carried out on 50 elderlies with mild cognitive impairment aged 65 80 living in rural areas of Kyungsangbuk-do. The study subjects haven't participated in other regular exercise programs (twice of week) during the past 6 months and the elderlies who received treatments for orthopedic ailment such as bone fracture within the last 6 months, had hearing and visual impairments, had neurological problems such as central nervous system damage, could not carry our exercise programs due to intellectual impairment or psychiatric defects, were taking drugs that can affect balancing ability, had ailments with circulatory organs such as heart were excluded. After explaining the purpose of this study, study methods, contents and procedures were sufficiently explained to all subjects, their agreements to participate in this study were received.

The study was carried out for 12 weeks between June 3, and August 24, 2013. The subjects participated in 24 sessions, twice each week, each lasting 50 minutes. The swiss ball program was conducted by two trainers and six assistants.

\section{Measurement}

Time up go: TUG, one leg standing balance test, tandem gait test, sit to stand test were conducted to evaluate lower extremity function.

\section{Procedures}

The Swiss Ball Program is made up of 24 sessions: two sessions each week and each session lasting 50 minutes and the each 50 minute session is made up of 10 minutes of warm up exercises, 30 minutes of main exercises, and 10 minutes of cool down exercises. The diameter of the Swiss Ball used was $55 \mathrm{~cm}$, selected after a consideration of the subjects' heights. The same stretching exercise routines were carried out for both the warm up and cool down to promote joint flexibility and in consideration of the subjects' fitness level, took a 5 minutes break between sets. Each exercise routine was carried out in three stages, from easy movement to difficult movement, and the ball exercise in lying down position, ball exercise sitting on the ball, and ball exercise using the wall were carried out.

\section{Data Analysis}

Using SPSS 12.0 for windows, the averages and standard deviations of all measured factors of the test results were calculated. In order to find out the statistical changes of timing of measurement (before and after carrying out the swiss ball program) between groups, an independent t-test was performed.

\section{RESULTS}

In order to find out what effects the swiss ball program has on the cognitive function, and low extremity function of the elderly with mild cognitive 
impairment, this study carried out the Swissball program for 12 weeks on the elderly with mild cognitive impairment(15 in the swiss ball program group and 15 in the control group). The results of the analysis of each factor are as follows:

\section{General Characteristics of Subjects}

The average age, height, and weight of the swiss ball program exercise group were 72 years, $155.2 \mathrm{~cm}$, and $61.7 \mathrm{~kg}$ respectively. The average age, height, and weight of the swiss ball program control group were 71 years, $153.3 \mathrm{~cm}$, and $62.4 \mathrm{~kg}$ respectively. There was not a significant difference between the two groups in the homogeneity test(Table 1).

Table 1. General characteristic of subjects

\begin{tabular}{ccrc}
\hline & Exp $(n=15)$ & Con $(n=15)$ & $p$ \\
\hline Age(year) & $72.11 \pm 4.86$ & $71.66 \pm 5.76$ & .369 \\
Height $(\mathrm{cm})$ & $155.21 \pm 8.52$ & $153.25 \pm 9.34$ & .607 \\
Weight(kg) & $61.66 \pm 5.12$ & $62.43 \pm 8.29$ & .704 \\
\hline
\end{tabular}

Exp: experimental group Con: control group

\section{Comparison of Changes in the Lower Extremity Function of the Swiss Ball Program Exercise Group and the Control Group}

The TUG of the swiss ball program exercise group before exercise session and after exercise session were $7.88 \pm 1.11$ and $6.28 \pm 0.59$ respectively, a significant difference $(\mathrm{p}<.05)$. In the control group, before exercise session and after exercise session were $8.23 \pm 1.43$ and $8.32 \pm 1.27$ respectively, an insignificant difference (p>.05)(Table 2).

Table 2. The comparison of time up and go withinsubjects at pre-post intervention

\begin{tabular}{lccc}
\hline & Pre & Post & $\mathrm{p}$ \\
\hline Experimental group & $7.88 \pm 1.11$ & $6.28 \pm 0.59$ & $.000^{*}$ \\
Control group & $8.23 \pm 1.43$ & $8.32 \pm 1.27$ & .627 \\
\hline *: P<.05 & & &
\end{tabular}

The one leg standing balance test of the Swiss ball program exercise group before exercise session and after exercise session were 10.88 \pm 5.34 and $18.70 \pm 1.68$ respectively, a significant differ- ence(p<.05). In the control group, before exercise session and after exercise session were 8.60 \pm 5.73 and $8.56 \pm 5.58$ respectively, an insignificant difference(p〉.05)(Table 3).

Table 3. The comparison of one leg standing balance test within subjects at pre-post intervention

\begin{tabular}{lrcc}
\hline & Pre & Post & $\mathrm{p}$ \\
\hline Experimental group & $10.88 \pm 5.34$ & $18.70 \pm 1.68$ & $.001^{* *}$ \\
Control group & $8.60 \pm 5.73$ & $8.58 \pm 5.58$ & .374 \\
\hline *: $\mathrm{P}<.05$ & & &
\end{tabular}

The tandem gait test of the swiss ball program exercise group before exercise session and after exercise session were $18.43 \pm 8.76$ and $14.67 \pm 9.23$ respectively, a significant difference( $p<.05)$. In the control group, before exercise session and after exercise session were $16.24 \pm 7.49$ and $17.57 \pm 6.89$ respectively, an insignificant difference $(\mathrm{p}\rangle .05)$ (Table 4).

Table 4. The comparison of tandem gait test within subjects at pre-post intervention

\begin{tabular}{lccc}
\hline & Pre & Post & $p$ \\
\hline Experimental group & $18.43 \pm 8.76$ & $14.67 \pm 9.23$ & $.009^{* *}$ \\
Control group & $16.24 \pm 7.49$ & $17.57 \pm 6.89$ & .309 \\
\hline *: $\mathrm{P}<.05$ & & &
\end{tabular}

The sit-to-stand test of the swiss ball program exercise group before exercise session and after exercise session were $15.20 \pm 2.33$ and $18.41 \pm 3.78$ respectively, a significant difference( $\mathrm{p}<.05)$. In the control group, before exercise session and after exercise session were $14.32 \pm 1.54$ and $14.77 \pm 1.32$ respectively, an insignificant difference(p>.05) (Table 5).

Table 5. The comparison of sit-to-stand test within subjects at pre-post intervention

\begin{tabular}{cccc}
\hline & Pre & Post & $p$ \\
\hline Experimental group & $15.20 \pm 2.33$ & $18.41 \pm 3.78$ & $.007^{* *}$ \\
Control group & $14.32 \pm 1.54$ & $14.77 \pm 1.32$ & .443 \\
\hline
\end{tabular}

*: $P<.05$ 


\section{DISCUSSION}

The weakening of basal physical fitness and decline in the ability to balance due to aging increase the risk of falls among the elderly and bring about not only a rise in medical cost but also a decline in the quality of life. Therefore, exercise programs of the elderly is being emphasized. The Swiss ball program employed in this study, a dynamic state exercise performed while body is resting on the unstable surface of the ball, is useful in strengthening muscles and improving the sense of balance by providing stability and dynamic mobility. It is also a suitable exercise for the elderly as it, compared to other muscle strengthening exercises, exerts less shock loads to the body and allows various movement freedom making it interesting to do(9). Therefore, it is intended to investigate the effects the Swiss ball program, on the elderly, 65 years or older, with mild cognitive impairment, twice a week for 12 weeks, has on the cognitive function and lower extremity function of the elderly with mild cognitive impairment.

The principle of the Swiss ball exercises is making a range of movements from small to large using working ranges of all joints. In particular, if a person with a pain in a specific area does the Swiss ball exercises, the pain area's movement and ability to adjust will improve. Doing the swiss ball exercises will improve not only muscle strength and endurance but also the ability to balance, flexibility and ability to adjust. Through the swiss ball exercises, prevention of the risk of elderly falling, improvement of the ability to balance, improvement of the functional fitness level, etc. can be achieved.

In this study, the cognitive function score significantly increased in the swiss ball program group, and in the control group, a significant change was not observed. When considering the cognitive function score drops on the average 3 points a year in regular dementia patients, the fact that there was a significant increase itself can be considered as meaningful.

Next, in this study, after the completion of the 12 weeks Swiss ball program, it was observed that there was a significant difference, and improvement, in the lower extremity function in the exercise group. When a group of female elderly participated in a 12 weeks aerobic dance exercise program, 3 sessions a week each session lasting 50 minutes, it was reported that the group's balance ability improved. The movements of the Swiss ball program invigorates the physiological stimulusresponse interaction of neuromuscular system. The gravity was reduced by the ball when doing the resting against the ball movement, despite the distance was the same. Thus, this allowed the subjects to improve their sense of balance by leading them to equilibrium reactions and difficult movement types in a relatively stable position $(10,11)$.

Through the results of preceding studies and this study, it has been shown that regular exercise can change the decline in the muscular strength and ability to balance due to aging(12). Thus the instability of the ball itself stimulates somatosensory and proprioceptive sensory functions to continuously maintain balance while exercising and rapidly invigorates the normally unused small muscles, used to maintain posture, and contributes towards improving the static'dynamic ability to balance. It is deemed that such improvements in the ability to balance lower the risk of falling by lowering the posture unrest and allow the elderly to enjoy a lifestyle that is more aggressive and active. Thus, as the swiss ball program has shown to be effective in improving the basal physical fitness and ability to balance of the elderly with mild cognitive impairment, it can be said that the Swiss ball program is an effective exercise program in preventing falls and promoting health. In addition, significant effects were observed in most other studies of exercise programs lasting from 8 weeks to 12 weeks or more. In this study, a significant improvement was observed after the exercise program lasting 12 weeks, 2 sessions each week. Through this, it has been confirmed that the exercise programs lasting 8 weeks or more using the Swiss ball improve the cognitive function and lower extremity function of the elderly with mild cognitive impairment.

\section{CONCLUSION}

This study conducted a swiss ball program, lasting for 12 weeks, 2 sessions per week for 50 minutes, on 30 elderlies with mild cognitive impairments and intended to find out the effects it has on lower extremity function. The results are as follows:

From the results of applying the swiss ball program on the elderly with mild cognitive impairment, 
it was observed that the TUG, one leg standing balance, tandem gait, sit-to-stand changes were significant in the Swiss ball program group $(\mathrm{p}<.01)$ and the Swiss ball program was effective in improving TUG, one leg standing balance, Tandem gait, sit-to-stand. Summarizing the above, the Swiss ball program, that can be implemented economically while doing interesting and fun exercises, has positive effects in improving the lower extremity function in the elderly with mild cognitive impairment in the coming aged society. Also, it is deemed that the Swiss ball program, as an active exercise intervention, is an effective means in improving the quality of life of the elderly with mild cognitive impairment.

\section{REFERENCES}

1. Aggarwal NT, Wilson RS, Beck TL. Motor dysfunction in mild cognitive impairment and the risk of incident Alzheimer disease. Arch neurol 2006; 63(12): 1763-1769.

2. Boyle PA, Wilson RS, Buchman AS. Lower extremity motor function and disability in mild cognitive impairment. Exp Aging Res 2007; 33(3): 355-371.

3. Thomas VS, Hageman PA. Can neuromuscular strength and function in people with dementia be rehabilitated using resi stance-exercise training? Results from a preliminary interv ention study. $J$ Science Med 2003; 5(8): 746-751.

4. Kent JA, Ng AV, Doyle JW. Human skeletal muscle responses vary with age and gender during fatigue due to incremental isometric exercise. $\mathrm{J}$ Appl Physiol 2002; 93(5): 1813-1823.

5. Flannery RB. Treating learned helplessness in the elderly dementia patient: preliminary inquiry. Am J Alzheimer's Demes 2002; 17(6): 346-349.

6. Toulotte C, Fabre C, Dangremont B. Effects of physical training on the physical capacity of frail demented patients with history of falling: a randomised controlled trial. Age Aging 2003; 32(1): 67-73.

7. Laurin D, Verreault R, Lindsay I, Macpherson K, Rockwood K. Physical activity and risk of cognitive impairment and elderly persons. Arch $\mathrm{Neu}^{-}$ rol 2001; 58(3): 498-504.

8. Cornillon E, Blanchon MA, Ramboa P, Braize C. Effectiveness of falls prevention strategies for elderly subjects who live in the community with performance assessment of physical act ivities(beforeafter). Med Phys 2002; 45(9): 493-503.

9. Teri L, McCurry SM, Buchner DM, Logsdon RG. Exercise and activity level in Alzheimer's disease: A potential treatment focus. J Rehavil Res Devel 1998; 35(4): 411-419.

10. Colcombe SJ, Erickson, KI, Raz N, Webb AG, Cohen NJ. Aerobic fitness reduce brain tissue loss in aging humans. J Gerontol Med 2003; 176180.

11. Cassilhas RC, Viana VA, Grassmann V, Santos R T, Santos RF, Tufik S, Mello MT. The impact of resistance exercise on the cognitive function of the elderly. Med Sci Sports Exerc 2007; 39(8): 1401-1407.

12. McDowell I. Alzheimer's disease: Insights from epidemiology. Aging(Milano) 2001; 13(3): 143-162. 